Cahiers de la recherche sur les droits

Cahiers

Fur les Droits fondamentaux

2 | 2003

Les titulaires particuliers des droits fondamentaux

\title{
Liberté d'expression et irresponsabilité des députés
}

Didier Baumont

\section{OpenEdition}

Journals

Édition électronique

URL : https://journals.openedition.org/crdf/7702

DOI : $10.4000 /$ crdf.7702

ISSN : 2264-1246

Éditeur

Presses universitaires de Caen

Édition imprimée

Date de publication : 1 janvier 2003

Pagination : $33-48$

ISBN : 2-84133-205-5

ISSN : 1634-8842

Référence électronique

Didier Baumont, «Liberté d'expression et irresponsabilité des députés », Cahiers de la recherche sur les droits fondamentaux [En ligne], 2 | 2003, mis en ligne le 18 décembre 2020, consulté le 14 novembre 2022. URL : http://journals.openedition.org/crdf/7702 ; DOI : https://doi.org/10.4000/crdf.7702 


\title{
Liberté d'expression et irresponsabilité des députés
}

\author{
Didier BAUMONT
}

Doctorant en droit public

ATER - Université de Caen Basse-Normandie

I. Les caractéristiques traditionnelles de l'irresponsabilité

A. L'étendue limitée de l'irresponsabilité

1. Les personnes concernées

2. Les actes visés

B. Les effets de l'irresponsabilité

II. La protection des fonctions parlementaires

A. La diversité des lieux de l'exercice des fonctions parlementaires

B. L'exercice des fonctions parlementaires

III. Les aménagements nécessaires

A. Les limites disciplinaires à l'irresponsabilité constitutionnelle

B. L'écart de situations entre l'Hémicycle et son perron

Gloire aux pays où l'on parle, honte aux pays où l'on se tait.

Georges Clemenceau devant la Chambre des députés, le 4 juin 1888.

Toute atteinte au dogme de l'irresponsabilité parlementaire porte en elle

le germe d'une régression du droit républicain, et partant, des libertés essentielles.

Michel Ameller, L'Assemblée nationale, Paris, PUF (Que sais-je?), 1994

La liberté d'expression des parlementaires s'inscrit dans les traditions historique et constitutionnelle françaises selon lesquelles les parlementaires doivent être protégés lorsqu'ils exercent leurs fonctions, au sein de l'enceinte délibérative. Cette protection ne doit certes pas être «interprété $[\mathrm{e}]$ comme un privilège d'impunité, allant à l'encontre de l'égalité de tous devant la loi ${ }^{1}$; un parle- mentaire court en effet toujours le risque d'être sanctionné disciplinairement, et l'irresponsabilité n'a pas une étendue illimitée. La protection dont jouissent les députés doit plutôt se définir comme une "immunité particulière ${ }^{2}$ prévue par l'article 26 de la Constitution, selon lequel « aucun membre du Parlement ne peut être poursuivi, recherché, arrêté, détenu ou jugé à l'occasion des

2. P. Avril, J. Gicquel, Lexique - Droit constitutionnel, Paris, PUF, 2001, p. 75 
opinions ou votes émis par lui dans l'exercice de ses fonctions». Pour O. Duhamel et Y. Mény, «l'immunité parlementaire recouvre deux garanties différentes: l'irresponsabilité et l'inviolabilité ${ }^{3}$. La différence entre les deux notions tient à ce que les domaines qu'elles définissent sont distincts. L'un affère à la liberté de parole des parlementaires; l'autre au fait que l'on ne puisse arrêter un parlementaire sans l'accord de son Assemblée, sauf cas de flagrant délit. Les deux protections représentent des immunités parlementaires ${ }^{4}$ qui « se rattachent intimement aux exigences primordiales du régime représentatif et au jeu normal des institutions dans les gouvernements constitutionnels ${ }^{5}$. La première relève de l'indépendance du parlementaire; la seconde, de l'entrave à l'encontre de l'exercice de ses fonctions ${ }^{6}$. Dans tous les cas, l'objectif de l'article 26 correspond à la logique de la séparation des pouvoirs telle qu'elle est pratiquée en France. Le but essentiel consiste à préserver les membres du Parlement de toutes poursuites judiciaires intempestives et injustifiées ${ }^{7}$.

Le fondement primordial de l'établissement de l'irresponsabilité, mais également de l'inviolabilité des représentants de la Nation, s'explique par la volonté de protéger le pouvoir délibérant, en tant que «pouvoir de vouloir ${ }^{8}$, en en garantissant l'indépendance ${ }^{9}$. Il s'agit d'une protection visant à prémunir les parlementaires contre tout ce qui pourrait nuire à la bonne marche des affaires publiques. Les personnes suspectées de pouvoir menacer cette indépendance doivent être comprises au sens large, et la protection doit trouver à s'appliquer à l'encontre de l'ensemble des particuliers mais aussi des membres du gouvernement qui pourraient chercher à étendre leur emprise sur les parlementaires. Ainsi, « dans les grands conflits politiques, un Gouvernement menacé pourrait être amené à se servir de la justice au profit de sa défense ou de ses rancunes ${ }^{10}$. La particularité majeure relative aux dirigeants, dans un système représentatif, est d'être responsables; et A. Esmein, lorsqu'il étudie la souveraineté nationale, expose les deux principales raisons de l'entorse faite à ce principe de responsabilité : «C'est tout d'abord que cette responsabilité est pratiquement très difficile à organiser. C'est que, d'autre part, elle tournerait le plus souvent contre l'intérêt public, contre le véritable intérêt des représentants, qui doit le plus souvent être libre pour être utile, en troublant ou interrompant le jeu continu des institutions représentatives ${ }^{11}$. On semble se diriger vers une protection de la double fonction du Parlement : légiférer et contrôler le Gouvernement sans entrave. Il ne s'agit donc pas d'une volonté d'organiser à tout prix une responsabilité, semblable à celle d'un particulier ; la situation dans laquelle se trouve le représentant de la Nation est évidemment de nature différente. La responsabilité du parlementaire ne peut être que politique et se manifester par un mandat de durée limitée. Les citoyens ne peuvent élire d'autres représentants que dans un climat et un moment propices, et non pas de façon incessante. Les principes du mandat impératif et de la révocabilité sont en effet bannis de nos régimes représentatifs ${ }^{12}$.

En 1943, J. Laferrière écrit, au sujet des immunités parlementaires, qu' « avec le degré auquel, dans la plupart des pays, atteignait la puissance parlementaire, leur justification paraît moins évidente. Le sentiment commun est souvent choqué de l'atteinte ainsi portée à l'égalité des individus devant la loi et devant la justice ${ }^{13}$. Aujourd'hui,

3. O. Duhamel, Y. Mény, Dictionnaire constitutionnel, Paris, PUF, 1992, p. 487. Voir également G. Cornu, Vocabulaire juridique, $3^{\mathrm{e}}$ éd., Paris, PUF, 2002, p. 493; "l'irresponsabilité est l'immunité au sens strict», p. 446. On utilisera désormais indifféremment les termes irresponsabilité et immunité, l'inviolabilité n'étant pas l'objet de cette étude. T.E. May, quant à lui, opère une différence, dans son Traité, entre d'une part le «privilège de la liberté de parole» et d'autre part «l'immunité d'arrestation ou de vexation». Voir T.E. May, Traité des lois, privilèges, procédures et usages du Parlement, Paris, Giard \& Brière, 1909, p. 101-109.

4. Voir par exemple dans ce sens G. Vedel qui affirme que les deux immunités parlementaires sont l'irresponsabilité et l'inviolabilité. Si l'irresponsabilité exonère de toute imputabilité du fait du dommage, G. Vedel prend soin d'ajouter que ceci n'est pas «incompatible [...] avec des sanctions disciplinaires d'ordre intérieur que le règlement de chaque assemblée prévoit à l'encontre de ses membres ». Voir G. Vedel, Manuel élémentaire de droit constitutionnel, Paris, Sirey, 1949, p. 402.

5. Les immunités parlementaires «tiennent à l'économie de la division des pouvoirs et au principe de la souveraineté législative. Elles sanctionnent pratiquement l'indépendance et la liberté du Parlement dans l'accomplissement de sa mission. Elles s'identifient en quelque sorte avec le droit de la nation de manifester sa volonté par l'organe de ses mandataires. Ce sont des prérogatives sans doute, c'est-à-dire des exigences supérieures de la vie gouvernementale; ce ne sont pas, à proprement parler, des privilèges, en tant que l'on entendrait par là des lois faites par des particuliers ou à leur bénéfice». Proposition de loi déposée par le sénateur belge M. Descamps le 4 avril 1900, rapportée par E. Pierre, Traité de droit politique, électoral et parlementaire, $2^{\mathrm{e}}$ éd., Paris, Librairies-Imprimeries réunies, 1902, rééd. Paris, Loysel, 1989, p. 1211.

6. P. Avril, J. Gicquel, Droit parlementaire, $2^{\mathrm{e}}$ éd., Paris, Montchrestien, 1996, p. 45.

7. G. Vedel, Manuel élémentaire de droit constitutionnel, p. 412. Le pouvoir disciplinaire ne consiste pas en des «attributions proprement pénales ou répressives que l'Assemblée exercerait, et qui d'ailleurs se heurteraient souvent à la règle de l'irresponsabilité, mais [en des] attributions proprement disciplinaires semblables à celles que connaissent le droit administratif ou le droit professionnel».

8. M. Prélot, Droit parlementaire français, Université de Paris, Institut d'études politiques (Les cours de droit), 1957-1958, t. II, p. 102.

9. J. Laferrière, Manuel de droit constitutionnel, Paris, Domat - Montchrestien, 1943, p. 661.

10. E. Pierre, Traité de droit politique..., p. 1210.

11. A. Esmein, Éléments de droit constitutionnel français et étranger, 6 éd., Paris, Sirey, 1914, rééd. Paris, LGDJ, 2001 , p. 440.

12. Voir par exemple J. Cadart, Le Régime électoral des États Généraux de 1789 et ses origines (1302-1614), Paris, Sirey (Annales de l’Université de Lyon), 1952, cité par P. Avril, «La naissance du droit parlementaire», in 1791, La Première Constitution française (Actes du colloque de Dijon, 26-27 septembre 1991), Paris, Economica (Droit public positif), 1993, p. 322 : «La répudiation du mandat impératif [...] fut entreprise par Louis XVI lui-même dans le règlement des élections du 24 janvier 1789, et confirmée par la Déclaration royale du 23 juin qui cessa les "restrictions de pouvoirs", parce que le concours attendu des États généraux exigeait que les députés ne fussent pas liés par les directives de leurs commettants mais capables de décider librement. »

13. J. Laferrière, Manuel de droit constitutionnel, p. 662. Cela fut particulièrement vrai en 1911, au sujet du rapport du député Viollette, relatif aux budgets locaux des colonies. 
force est de constater que cette immunité n'est pas, ou plus, décriée par l'opinion publique. Le dernier débat public a concerné l'immunité juridictionnelle et temporaire du chef de l'État, mais pas les immunités des parlementaires. De leur côté, les ministres sont loin de demander pareille immunité et acceptent d'être poursuivis pour diffamation en raison des propos qu'ils tiendraient pendant l'exercice de leurs fonctions. Ce fut d'ailleurs récemment le cas, en mai 2000, pour S. Royal, alors ministre déléguée à l'Enfance et à la Famille, qui dut comparaître devant la Cour de justice de la République pour « complicité de diffamation envers des fonctionnaires publics» et qui fut acquittée.

Les immunités dont bénéficient députés et sénateurs visent donc à ce que leurs travaux ne soient pas entravés par les citoyens, et indirectement par les juges, mais également par le Gouvernement. E. Pierre ne dit-il pas qu' « une inviolabilité plus nécessaire encore que celle qui protège la personne en cas d'accusation de crimes ou délits de droit commun est celle qui protège la salle des délibérations, qui couvre les paroles et les actes des représentants du pays ${ }^{14}$ ? Selon O. Beaud, "la question de savoir si telle ou telle opinion est couverte par cette irresponsabilité, dépend du lieu où elle a été prononcée ${ }^{15}$. J. Laferrière, quant à lui, écrit que « le rôle des assemblées modernes est essentiellement un rôle de contrôle et de critique. Elles ne peuvent le remplir efficacement que si chaque membre du Parlement est individuellement assuré d'une complète indépendance ${ }^{16}$. Protection du rôle du Parlement et protection des membres qui le composent semblent donc être intimement liées. Mais quelle est exactement la nature de ce lien ? Est-ce originellement la fonction qui bénéficie de cette immunité particulière, ou bien simplement le fait de s'exprimer dans l'enceinte parlementaire? Deux situations peuvent se distinguer. Dans le premier cas, le député s'exprime - débat, vote, etc. - dans le cadre de ses fonctions; dans le second, il ne fait que s'exprimer dans l'enceinte parlementaire. Pourtant, on pouvait lire encore récemment dans la doctrine ${ }^{17}$ un amalgame entre les deux situations précitées : l'irresponsabilité des propos et actes du parlementaire dans l'exercice de ses fonctions signifie que cette immunité
« confère à ses titulaires une totale liberté de parole dans l'enceinte parlementaire ${ }^{18}$.

L'ambiguïté des textes constitutionnels successifs portait sur le sujet de cette protection : le parlementaire qui exerce ses fonctions, ou le lieu de cet exercice? Si la mission des représentants de 1789 apparaît comme définie, l'évolution et la diversification contemporaines du travail parlementaire viennent compliquer la situation d'alors.

Les origines écrite et historique de l'irresponsabilité parlementaire sont britanniques, comme nous le rappelle A. Esmein. Plus précisément, elles sont incluses dans le Bill of Rights qui affirme que «la liberté de la parole, des débats et des procédures dans le Parlement ne pourrait être l'objet d'une poursuite ou être mise en question devant aucune cour ou dans aucun lieu en dehors du Parlement ${ }^{19}$. Aujourd'hui, l'irresponsabilité n'est toutefois pas « codifiée explicitement ${ }^{20}$ en Angleterre.

L'origine française de cette protection, sans cesse reprise par les Constitutions successives, est un des premiers principes de la période révolutionnaire: «le jour même où notre première Assemblée nationale entra en lutte ouverte avec la Cour, le 23 juin 1789, elle décréta que "la personne de chacun des députés est inviolable" " ${ }^{21}$. A l'occasion de cette décision, l'Assemblée nationale ajoute "que tous particuliers, toute corporation, tout tribunal, cour ou commission qui oseraient, pendant ou après la présente session, poursuivre, rechercher, arrêter ou faire arrêter, détenir ou faire détenir un député pour raison d'aucune proposition, avis, opinion ou discours faits par lui aux États Généraux [...], sont infâmes et traîtres envers la nation, et coupables de crime capital ${ }^{22}$. Ce souci de précision et de menace semble aujourd'hui quelque peu atténué, et dès l'article 7 de la section $\mathrm{V}$ de la Constitution du 3 septembre 1791, la formulation devient plus brève et plus directe ${ }^{23}$. Le choix des constituants de 1791 se porte sur l'exercice des fonctions parlementaires plutôt que sur celui de l'enceinte parlementaire. Mais la Constitution suivante, en date du 24 juin 1793, va préférer retenir, quant à elle, l'enceinte ${ }^{24}$. Dès lors, trois périodes constitutionnelles vont se succéder, pendant lesquelles vont alterner les deux conceptions. La première d'entre

14. E. Pierre, Traité de droit politique..., p. 1259

15. O. Beaud, "Ordre constitutionnel et ordre parlementaire; une relecture à partir de Santi Romano », Droits, n 33, $2001, \mathrm{p} .88-89$.

16. J. Laferrière, Manuel de droit constitutionnel, p. 661-662.

17. S. Depré, «La liberté d'expression, la presse et la politique», Revue belge de droit constitutionnel, 2001, p. 375 sq.; et surtout p. 381 sq. Il s'agissait en l'espèce de l'article 58 de la Constitution belge, mais ce dernier ne diffère dans sa rédaction que très peu de l'article 26 de notre Constitution; seul l'énoncé est un peu moins précis.

18. Ibid., p. 381. C’est nous qui soulignons.

19. Cité par A. Esmein, Éléments de droit constitutionnel..., p. 954.

20. «Les immunités parlementaires. Rapport définitif de M. Robert Myttenaere », Informations constitutionnelles et parlementaires, $\mathrm{n}^{\circ}{ }^{0} 175,1998, \mathrm{p} .121$.

21. Il faut entendre ici la future irresponsabilité, différente de l'inviolabilité. Cette dernière ne sera vraiment définie qu'un an plus tard, le 26 juin 1790. Voir E. Pierre, Traité de droit politique..., p. 1203.

22. Le décret se termine, nous dit E. Pierre, par: «L'Assemblée nationale arrête que, dans les cas susdits, elle prendra toutes les mesures nécessaires pour faire rechercher, poursuivre et punir ceux qui en seront les auteurs, instigateurs ou exécuteurs.» Voir E. Pierre, Traité de droit politique..., p. 1259.

23. Article 7, section V « Réunion des représentants en Assemblée nationale législative», de la Constitution du 3 septembre 1791 : «Les représentants de la Nation sont inviolables : ils ne pourront être recherchés, accusés ni jugés en aucun temps pour ce qu'ils auront dit, écrit ou fait dans l'exercice de leurs fonctions de représentants. »

24. Article 43 de la Constitution du 24 juin 1793: «Les députés ne peuvent être recherchés, accusés et jugés en aucun temps, pour les opinions qu'ils ont énoncées dans le sein du Corps législatif.» 
elles comprend les Constitutions de l'An III ${ }^{25}$ et de l'An VIII ${ }^{26}$. Ces deux textes retiennent uniquement l'exercice des fonctions et semblent abandonner l'élément spatial. De plus, on peut remarquer que la référence au terme d'irresponsabilité fait son entrée dans le texte constitutionnel de 1799.

Ensuite, la seconde période constitutionnelle se compose des Chartes de 1814 et 1830, ainsi que des Constitutions de 1848 et 1852 . Sous les monarchies constitutionnelles, c'est l'élément spatial qui est effectivement retenu. On peut remarquer que l'irresponsabilité n'est pas organisée dans le texte constitutionnel même, mais dans la loi du 17 mai $1819^{27}$. Certes, le corps des Chartes ne contient pas cette immunité parlementaire, néanmoins, la rédaction du texte gagne désormais en précision par rapport aux Constitutions précédentes, sans toutefois réitérer la rédaction de 1789. Et il est important de noter que pour la première fois, on va organiser le statut des comptes rendus des séances. En l'occurrence, la double condition de la fidélité de l'écrit et de la «bonne foi » de son auteur est posée. Avec la Constitution de la II ${ }^{\text {nde }}$ République, l'irresponsabilité est de nouveau présente dans le corps du texte constitutionnel, et on s'inscrit toujours dans la lignée de la rédaction de 1793 et des deux Chartes, c'est-à-dire le choix de l'élément spatial ${ }^{28}$. Il en va de même sous le Second Empire. Toutefois, ce régime présente deux particularités en la matière. En effet, la Constitution de 1852 ajoute une précision importante relative à la nature des comptes rendus de séances : ceux-ci deviennent désormais officiels par disposition constitutionnelle ${ }^{29}$. Mais dans un autre temps, l'irresponsabilité des parlementaires n'est plus inclue dans le corps du texte constitutionnel, à la manière des Chartes.
C'est un décret organique du 2 février 1852 qui vient préciser cette immunite ${ }^{30}$.

Enfin, vient la troisième, et pour l'instant dernière, période constitutionnelle : les $\mathrm{III}^{\mathrm{e}}, \mathrm{IV}^{\mathrm{e}}$ et $\mathrm{V}^{\mathrm{e}}$ Républiques. Ces trois Républiques retiennent l'exercice des fonctions parlementaires dans leurs articles afférant à l'irresponsabilité des députés ${ }^{31}$. On peut apprécier que les rédacteurs de 1946 aient préféré une rédaction plus complète que celle de 1875 et que ceux de 1958 l'aient adoptée, ne cherchant pas à opérer, sur ce point, une rupture avec le texte précédent.

L'affaire semble entendue et la plupart des manuels contemporains de droit constitutionnel ne font que peu de cas de la notion d'irresponsabilité parlementaire, ne lui consacrant au plus que quelques lignes de développement $^{32}$. Dans cette situation, on ne peut que louer la volonté d'O. Beaud de relancer le débat en expliquant que l'irresponsabilité porte, même actuellement, davantage sur l'enceinte parlementaire que sur l'exercice des fonctions et la personne du député ou du sénateur ${ }^{33}$. L'auteur de La Puissance de l'État se fonde notamment sur les écrits d'E. Pierre qui affirme par ailleurs qu' « une autre inviolabilité ${ }^{34}$ protège l'enceinte du Parlement ${ }^{35}$. La remise en cause du sujet de l'irresponsabilité parlementaire ne semble donc pas être sans fondement. En effet, les fonctions du parlementaire sous la III $^{\mathrm{e}}$ République ne recouvrent pas exactement les mêmes que celles d'aujourd'hui. Et si la double fonction du Parlement demeure de légiférer et contrôler l'action du Gouvernement, il n'en va pas de même des fonctions du parlementaire. Celui-ci a vu son rôle se diversifier sous la $\mathrm{V}^{\mathrm{e}}$ République, allant de la saisine du Conseil constitutionnel à celle du Médiateur de la République, par exemple ${ }^{36}$.

25. Article 110 de la Constitution du 5 fructidor An III : «Les citoyens qui sont, ou ont été, membres du Corps législatif, ne peuvent être recherchés, accusés, ni jugés en aucun temps, pour ce qu'ils ont dit ou écrit dans l'exercice de leurs fonctions.»

26. Article 69 de la Constitution du 22 frimaire An VIII : «Les fonctions des membres soit du Sénat, soit du Corps législatif, soit du Tribunat, celles des consuls et des conseillers d'État ne donnent lieu à aucune responsabilité.»

27. On peut reprendre ici la citation de la loi du 17 mai 1819 qu'en fait E. Pierre: «Art. 21 - Ne donneront ouverture à aucune action, les discours tenus dans le sein de l'une des deux Chambres, ainsi que les rapports ou toutes autres pièces imprimées par ordre de l'une des deux Chambres. Art. $22-\mathrm{Ne}$ donnera lieu à aucune action, le compte fidèle des séances publiques de la Chambre des députés, rendu de bonne foi dans les journaux.» De plus, E. Pierre ajoute à ces deux articles que «sous la Restauration et la monarchie de Juillet, à une époque où les Chambres ne publiaient aucun compte rendu officiel de leurs séances, les jurisconsultes inféraient de ces mots: “Discours tenus dans le sein de l'une des deux Chambres”, qu'il pourrait y avoir action publique ou privée contre le pair ou le député, ou contre le tiers qui, sans l'ordre de la Chambre, aurait reproduit, en dehors de son enceinte, le discours qui y aurait été prononcé (V. Chassan, Des délits de la parole, I, p. 57). »Voir E. Pierre, Traité de droit politique..., p. 1263.

28. Article 36 de la Constitution du 4 novembre 1848 : «Les représentants du peuple sont inviolables. Ils ne pourront être recherchés, accusés, ni jugés, en aucun temps, pour les opinions qu'ils auront émises dans le sein de l'Assemblée nationale."

29. Article 42 de la Constitution du 14 janvier 1852 : «Le compte rendu des séances du Corps législatif par les journaux ou tout autre moyen de publication, ne consistera que dans la reproduction du procès-verbal, dressé, à l'issue de chaque séance, par les soins du président du Corps législatif.»

30. Décret organique du 2 février 1852 : «Les députés ne pourront être recherchés, accusés ni jugés en aucun temps pour les opinions qu’ils auront émises dans le sein du Corps législatif. »

31. Article 13 de la loi constitutionnelle du 16 juillet 1875 : «Aucun membre de l'une ou de l'autre Chambre ne peut être poursuivi ou recherché à l'occasion des opinions ou votes émis par lui dans l'exercice de ses fonctions. » Article 21 de la Constitution du 27 octobre 1946: «Aucun membre du Parlement ne peut être poursuivi, recherché, arrêté, détenu ou jugé à l'occasion des opinions ou votes émis par lui dans l'exercice de ses fonctions. »

32. Voir, par exemple, P. Pactet, Institutions politiques - Droit constitutionnel, Paris, Armand Colin, 2002, p. 462-463. Voir également F. Hamon, M. Troper, Droit constitutionnel, $27^{\mathrm{e}}$ éd., Paris, LGDJ, 2001, p. 607-608.

33. "C'est donc bien le lieu qui trace le moment de démarcation du droit de l'irresponsabilité» (O. Beaud, "Ordre constitutionnel et ordre parlementaire... », p. 89). La démonstration d'O. Beaud semble a priori en contradiction avec ce qu'écrit T. S. Renoux: «l'irresponsabilité [...] tend à protéger la fonction parlementaire et la personne même du parlementaire ", sans aucune référence au lieu d'expression de l'opinion. Voir T. S. Renoux, «Immunité et parlementaires : plaidoyer pour une cause défunte», RFDC, 1990, p. 247.

34. Comprendre ici l'acception actuelle de l'irresponsabilité.

35. E. Pierre, Traité de droit politique..., p. 1211. Cela laisserait présager que l'ambiguïté perdure depuis la III République.

36. T. S. Renoux, «Immunité et parlementaires... », p. 251 sq. L'auteur y explique notamment que "d'une manière générale, la fonction parlementaire n'est pas assimilable à la fonction législative. L'une concerne le député ou le sénateur, l'autre le Parlement. La fonction parlementaire recouvre une réalité non seulement constitutionnelle mais également législative, voire réglementaire (telle que fixée par les règlements intérieurs des assemblées [...]». 
En outre, les lieux de l'exercice des fonctions sont devenus multiples. Le Palais-Bourbon, s'il demeure le haut lieu de l'action parlementaire, n'en est plus l'endroit exclusif. Fonctions et lieux d'exercice ne peuvent plus être définis aussi facilement que sous les régimes précédents. On peut alors s'interroger sur le fait que le régime de l'irresponsabilité, lui, n'a pas changé, ne bénéficiant pas, comme celui de l'inviolabilité, d'une révision constitutionnelle ${ }^{37}$. Il semble donc utile d'examiner la nature de l'irresponsabilité des députés (I) pour mieux en redéfinir l'objet réel (II) et se convaincre de la nécessité de certains aménagements (III).

\section{Les caractéristiques traditionnelles de l'irresponsabilité}

L'étendue de l'irresponsabilité parlementaire se trouve limitée (A), et ses effets, généralement sans nuances, se prolongent jusqu'aux comptes rendus (B).

\section{A. L'étendue limitée de l'irresponsabilité}

L'expérience française n'a pas connu l'évolution anglaise, pluriséculaire. Elle s'en est plutôt fortement inspirée. Les constituants des premières Constitutions françaises ont adopté, sur bon nombre de points, la solution aboutie des Britanniques. Ce sont en effet les membres de la Chambre des communes qui ont eu l'occasion, les premiers, de s'atteler à définir l'étendue de cette immunité $^{38}$.

\section{Les personnes concernées}

Les immunités parlementaires, et l'irresponsabilité en particulier, sont au centre des conditions de l'exercice normal de tout régime représentatif. Allant dans ce sens, la Cour de cassation reprend, en 1879, les observations du rapporteur général R. de Chenevières, selon lequel «soit la Chambre des députés, soit le Pouvoir exécutif, font partie des pouvoirs constitués dans lesquels l'exercice de la souveraineté se résume, et qu'à moins de substituer la confusion à la séparation des pouvoirs, base de notre régime politique, il ne peut appartenir à aucun tribunal de les traduire indirectement à sa barre, en leur demandant compte de leurs agissements ${ }^{39}$. Tout d'abord, l'irresponsabilité bénéficie, bien entendu, aux membres des assemblées parlementaires. Par conséquent, c'est la qualité intrinsèque de député ou de sénateur qui octroie cette protection. Les textes constitutionnels des trois dernières Républiques sont précis à ce sujet. Toutefois, la loi du 29 juillet 1881 diffère quelque peu, dans sa rédaction, de la loi constitutionnelle du 16 juillet 1875 . L'article $41^{40}$ de la loi de 1881 est effectivement moins précis, et cela a pour effet pour la compréhension d'élargir le nombre de personnes, auteurs de discours, couvertes par l'irresponsabilité parlementaire. De cette façon, le principe n'est plus exactement le même puisque «ce n'est pas la personne qui a tenu les propos qui est prise en considération, mais les propos tenus, en tant qu'ils sont un élément des discussions parlementaires ${ }^{41}$. Il est tout à fait possible d'admettre que les deux textes se complètent et que « ne pas appliquer l'alinéa premier de l'article 41 de la loi du 29 juillet 1881 à des personnes étroitement impliquées dans des activités liées, de manière indubitable, à l'exercice du mandat de parlementaire, reviendrait à lui retirer toute portée pratique réelle ${ }^{42}$. S'ajoute alors la question du statut des dépositions recueillies par les commissions d'enquête parlementaires; en effet, celles-ci sont habituellement amenées à auditionner des personnes qui ne sont pas des parlementaires. Ces dépositions bénéficient-elles de la même protection dont dispose la déclaration d'un député ? Le problème est sensible, et sa première résolution juridictionnelle laissait entrevoir une situation peu favorable pour la personne auditionnée. La Cour d'appel de Bordeaux, le 26 juillet $1878^{43}$, provoqua des réactions à

37. $\mathrm{LC}^{\circ}$ 95-880 du 4 août 1995 portant extension du champ d'application du référendum, instituant une session parlementaire ordinaire unique, modifiant le régime de l'inviolabilité parlementaire et abrogeant les dispositions relatives à la Communauté et les dispositions transitoires, JO, 5 août 1995, p. 11744 .

38. On trouve l'origine de la consécration de l'irresponsabilité peu après l'avènement d'Henry IV, grâce à la volonté de celui-ci et à l'unanimité des membres de la Chambre des lords. À cette époque, la Chambre des communes adressa une pétition au roi, se plaignant d'une violation des coutumes parlementaires, et en particulier de la «freedom of speech». Le roi et la Chambre des lords consentirent alors à reconnaître ce nouveau "privilège" aux Communes. La conséquence finale de cette pétition fut que les représentants des Communes purent désormais aller jusqu'à critiquer, dans la limite de l'exercice de leurs fonctions, les décisions royales. Toutefois, si ce principe est définitivement posé en Angleterre dès cette époque, le droit parlementaire étant un droit éminemment coutumier (P. Avril, J. Gicquel, Droit parlementaire, p. 3), l'étendue de l'immunité de parole fit l'objet de bon nombre d'éclaircissements, dont un notable en 1621. À cette date, en effet, la Chambre des communes est amenée à définir l'étendue de cette immunité. T.E. May nous rapporte que la Chambre basse déclare « que chaque membre a la liberté, à l'abri de tout obstacle, de tout emprisonnement ou de toute vexation, sauf la censure de la Chambre elle-même, pour ou touchant tout bill, de parler, de raisonner ou faire une déclaration sur toute affaire ou affaire regardant le Parlement ou les affaires parlementaires. » Ce principe fut solennellement confirmé le 11 décembre 1667. Enfin, lors de la Révolution, cette immunité parlementaire fut inscrite en 1689 dans l'article $9 \mathrm{du}$ Bill of Rights. Mais cette reconnaissance, aussi formelle et importante fut-elle, ne fit pas l'économie de précisions importantes quant à son étendue. En effet, si l'accord entre le roi et les deux Chambres portait sur la reconnaissance de ce privilège, c'est sur son étendue que se posa le problème. À ce sujet, les parlementaires eurent l'opportunité, à l'occasion d'affaires célèbres, de définir le champ d'application de cette liberté de parole. Voir T.E. May, Traité des lois..., p. 103 sq. Voir également H. Isar, «Immunités parlementaires ou impunité du parlementaire?», RFDC, 1994, p. 683-684.

39. Observations citées par E. Pierre, Traité de droit politique..., p. 1270.

40. «Ne donneront ouverture à aucune action les discours tenus dans le sein de l'Assemblée nationale ou du Sénat ainsi que les rapports ou toute autre pièce imprimée par ordre de l'une de ces deux assemblées ».

41. J. Laferrière, Manuel de droit constitutionnel, p. 666.

42. J. Desandre, «La responsabilité des personnes entendues par les commissions parlementaires d'enquête », RDP, 1986, p. 733.

43. Deux arrêts : CA Bordeaux, 26 juillet 1878, Labadie c. Moreau, Sirey, 1879, II, p. 226. 
l'encontre des deux arrêts qu'elle rendit ce jour-là ${ }^{44}$. Alors que l'accusé estimait être couvert par une immunité "pour les expressions incriminées» devant recevoir la qualité de «déposition devant les membres de la Commission », la Cour affirma que "l'immunité ne s'applique qu'à la personne qui a juridiquement la qualité de témoin ", qualité dont seule l'autorité judiciaire dispose ${ }^{45}$. Deux ans plus tard, la Chambre des députés se prononce sur cette délicate question et affirme très clairement sa position par le biais de l'une de ses commissions: «La Commission se considère comme investie d'un mandat judiciaire; elle entendra donc les témoins sous la foi du serment; ces témoins seront couverts comme tous ceux qui déposent devant la justice ${ }^{46}$. À cette date, il semble évident que les députés exercent pleinement les droits que l'autonomie des assemblées parlementaires peut leur octroyer, allant jusqu'à contredire une décision de justice lorsqu'ils estiment qu'il leur revient d'interpréter la Constitution et le règlement de la Chambre des députés. En 1953, une loi vient tenter de résoudre ce problème: «jusqu'au dépôt du rapport général d'une commission d'enquête ", le secret est obligatoire et toute publication, interdite ${ }^{47}$. Ce n'est qu'en 1983 que cette loi reçut une première application jurisprudentielle ${ }^{48}$; les juges des premier et second ressorts déclarant l'action irrecevable ${ }^{49}$, et accordant "une portée [...] restrictive aux dispositions de la loi du 29 juillet $1881{ }^{50}$. Le principe du secret des auditions fut abandonné avec la loi organique du 20 juillet $1991^{51}$ qui rétablit la publicité des travaux parlementaires afin d'éviter des pratiques arbitraires. En effet, « la pratique variait selon les commissions » et «il est à noter qu'aucune poursuite ni sanction n'était intervenue, en dépit des fuites qui se produisirent à plusieurs reprises ${ }^{52}$ alors que la règle du secret devait s'appliquer tant que les travaux n'étaient pas terminés. En outre, la pratique du secret du rapport divergeait entre le Sénat et l'Assemblée nationale, et même entre les commissions au sein de cette dernière, certaines publiant l'intégralité des auditions, d'autres pas ${ }^{53}$.

\section{Les actes visés}

«L'immunité qui couvre les paroles et les votes des représentants du pays ne permet pas que les actes du Sénat ou de la Chambre des députés soient appréciés par les tribunaux; ces actes échappent à la compétence de toutes les juridictions, parce que les deux Chambres font partie des pouvoirs publics dans l'ensemble desquels réside l'exercice de la souveraineté $"{ }^{54}$. L'irresponsabilité parlementaire profite à certains types d'actes : classiquement, il s'agit des opinions, votes, rapports ${ }^{55}$, propositions de loi émanant des parlementaires ainsi que, plus généralement, de tout document imprimé sur ordre des Chambres. Ainsi, les questions écrites, publiées au Journal officiel, bénéficient également de l'irresponsabilité. J. Lyon relate un précédent démontrant que les députés entendaient encore exercer pleinement le principe d'autonomie des Chambres, sous la $\mathrm{IV}^{\mathrm{e}}$ République. Selon ce précédent, le Président Le Troquer affirma l'effectivité de la protection relative aux questions écrites, estimant que l'interprétation qu'il faisait des dispositions réglementaires allait en ce sens ${ }^{56}$.

\section{B. Les effets de l'irresponsabilité}

«Les poursuites sont interdites s'il s'agit d'attaquer le parlementaire pour les opinions ou votes émis par lui dans l'exercice de ses fonctions ; le parlementaire est de ce point de vue irresponsable. Dans les autres cas, le parlementaire n'est pas irresponsable, mais on considère que sa personne est inviolable ${ }^{57}$. L'irresponsabilité parlementaire a pour effet d'écarter toute poursuite disciplinaire ${ }^{58}$, pénale ou civile, même si cela doit aller à l'encontre du principe de l'article 1382 du Code civil. «Pour garantir pleinement son indépendance», explique A. Esmein, «pour écarter les

44. Voir les remarques de J.-E. Labbey, Sirey, 1879, II, p. 225-226.

45. Trib. corr. Blaye, Labadie c. Moreau, Sirey, 1879, II, p. 226.

46. Cité par E. Pierre, Traité de droit politique..., p. 693-694. C'est nous qui soulignons.

47. Articles $1^{\text {er }}$ et 2 de la loi $n^{\circ}$ 53-1215 du 8 décembre 1953 relative au secret des travaux des commissions d'enquête parlementaires, JO, 9 décembre 1953 , p. 10943. Cette loi fut adoptée en réaction à l'affaire Général Revers, en 1950, relative à une déposition faite devant une commission d'enquête. Voir Gazette du Palais, 1956, Jurisprudence, p. 375-376.

48. TGI Paris, 5 mai 1983, Orvain-Creff c. Deleplace, puis CA Paris, 11 janvier 1984, Orvain-Creff c. Deleplace. Cités par J. Desandre, «La responsabilité des personnes... », p. 733-734.

49. «Par application des dispositions de l'article 41, alinéa premier de la loi du 29 juillet [1881] sur la liberté de la presse». Voir J. Desandre, «La responsabilité des personnes... ", p. 731 sq.: «La règle du secret constitue l'élément déterminant qui exonère en fait les témoins de toute responsabilité, pour les déclarations formulées devant une commission d'enquête.»

50. G. Bergougnous, «Le statut de parlementaire. De l'application souveraine à la souveraineté du droit», RDP, nº spécial, 2002, p. 342.

51. LO n ${ }^{\circ}$ 91-698 du 20 juillet 1991, tendant à modifier l'article 6 de l'ordonnance n ${ }^{\circ}$ 58-1100 du 17 novembre 1958 relatif aux commissions d'enquête et de contrôle parlementaires, JO, 23 juillet 1991, p. 9727. Exceptionnellement, la commission peut tout de même décider d'organiser des auditions sous le sceau du secret.

52. P. Avril, J. Gicquel, Droit parlementaire, p. 246.

53. Ibid.

54. E. Pierre, Traité de droit politique..., p. 1268.

55. R. Bonnard présenta la «lacune» consistant à ne pouvoir engager des poursuites pour des fautes commises à l'occasion des rapports parlementaires. Mais l'auteur en arrivait à la conclusion qu'il ne s'offrait comme solution qu'à entreprendre « une œuvre de moralisation ». Voir R. Bonnard, "Immunités et discipline parlementaires à l'occasion des rapports des commissions», RDP, 1911, p. 558 sq., et surtout p. 562 sq.

56. J. Lyon, Nouveaux suppléments, t. II, Paris, Assemblée nationale, 1990, p. 620.

57. E. Zoller, Droit constitutionnel, $2^{\mathrm{e}}$ éd., Paris, PUF, 1999, p. 363.

58. Hormis le pouvoir de police reconnu au président de l'Assemblée nationale. 
poursuites à raison de délits imaginaires, il a paru nécessaire de soustraire à l'action de la loi même les délits réels, qui peuvent être contenus dans l'acte par lequel il exerce sa fonction. Comme il arrive plus d'une fois, la protection n'a paru efficace qu'à la condition d'être absolue » ${ }^{59}$. Contrairement au modèle britannique, l'irresponsabilité française a historiquement un caractère absolu. En effet, la « freedom of speech» dont bénéficient les parlementaires d'outreManche n'a qu'un caractère relatif. Ainsi, T.E. May rappelle que «d'après la coutume ancienne autant que d'après la loi, un membre ne [peut] être interrogé en dehors du Parlement, [par contre] il peut être censuré et puni par la Chambre même dont il est membre. [...] Quelques membres ont été admonestés, d'autres incarcérés et, aux Communes, quelques-uns expulsés " ${ }^{60}$. Historiquement, ce sont les Chambres anglaises qui apprécient les limites à l'application de la liberté de parole des parlementaires, décidant au cas par cas, le cas échéant, l’incompétence des juges. Cela participe de l'autonomie classique des Chambres, mais aussi et surtout de la pondération des parlementaires britanniques, dont on reconnaît la capacité à se modérer par eux-mêmes. L'exemple suisse s'inscrit dans cette même optique puisque l'irresponsabilité signifie que « le député peut abreuver d'injures ses collègues ou des tiers, il peut faire planer sur eux les soupçons les plus diffamatoires, sans encourir de sanction ni pénale, ni civile, ni disciplinaire (le rappel à l'ordre excepté, selon, l'article 32 du règlement du Conseil national, du 2 octobre 1962). On le voit, le privilège est de taille; mais il est heureusement compensé par la placidité ${ }^{61}$ de nos hommes politiques » ${ }^{62}$.
À la sagesse des Chambres britanniques, les constituants français ont préféré substituer un caractère absolu à l'irresponsabilité parlementaire. En France, l'irresponsabilité revêt un caractère d'ordre public. Cette immunité parlementaire n'est donc pas d'ordre privé, ce qui signifie que les parlementaires ne peuvent pas demander à ce qu'elle soit levée. «Le parlementaire», affirme J. Laferrière en 1943, «ne peut renoncer à son irresponsabilité. L'engagement qu'il prendrait de ne pas s'en prévaloir en cas de poursuites dirigées contre lui serait sans valeur. L'exception d'irresponsabilité peut être invoquée en tout état de cause, même pour la première fois en cassation. Le juge doit l'opposer d'office ${ }^{63}$. Plaidant pour une irresponsabilité absolue, G. Vedel ajoute: «aucune autorité, à commencer par l'Assemblée intéressée, n'a qualité pour lever ou suspendre l'irresponsabilité » ${ }^{64}$. Un corollaire au caractère absolu de l'irresponsabilité est sa perpétuité: «à aucun moment le parlementaire ne pourra être poursuivi, alors même qu'il aura cessé d'être député ${ }^{65}$. On retrouve ainsi la justification de la qualification d'immunité à l'irresponsabilité puisque « la situation particulière de l'auteur [en l'espèce le fait qu'il soit député] de l'infraction au moment où il commet celle-ci, s'oppose définitivement à toute poursuite, alors que la situation créant ce privilège a pris fin ${ }^{66}$.

Les comptes rendus des débats parlementaires, rapportés dans les journaux, ne furent pas un problème simple à résoudre. Suivant les régimes, le statut des comptes rendus a évolué, et la jurisprudence leur étant relative a dû faire de même ${ }^{67}$. Le problème juridique se focalisa tout

59. A. Esmein, Éléments de droit constitutionnel..., p. 955.

60. T.E. May, Traité des lois..., p. 105.

61. C'est nous qui soulignons.

62. J.-F. Aubert, Traité de droit constitutionnel suisse, t. II, Neuchâtel, Ides et Calendes, 1967, p. 467.

63. J. Laferrière, Manuel de droit constitutionnel, p. 667-668.

64. G. Vedel, Manuel élémentaire de droit constitutionnel, p. 402.

65. J. Laferrière, Manuel de droit constitutionnel, p. 667.

66. G. Cornu, Vocabulaire juridique, p. 445.

67. Il y a de cela un siècle et demi, la Cour de cassation, dans un arrêt du 8 février 1850 (Cass., 8 février 1850, Le Siècle et Le National c. Ségur d'Aguesseau, Dalloz, 1850, I, p. 70), apporta une précision quant à la portée de la protection octroyée aux journaux. À cette époque, où les comptes rendus des journalistes étaient encore " arbitraires " (ils ne deviendront officiels qu'avec la Constitution de 1852, cf. note 29), un député fut cité dans deux journaux à l'occasion d'un discours qu'il avait prononcé à la tribune. Le Siècle et Le National n'acceptèrent pas sa demande de droit de réponse; le député exigeait que la totalité du débat soit reproduit. L'affaire fut portée devant les tribunaux, qui donnèrent raison au député, en première instance et en appel. Les deux journaux se pourvurent en cassation, et on eut alors droit aux premières précisions au sujet du régime particulier réservé aux comptes rendus publiés dans les journaux. L'avocat général Plougoulm précisa la portée de l’article 22 de la loi du 17 mai 1819 (cf. note 27) en estimant que « sans doute, dans le but d'assurer aux séances de nos assemblées toute la publicité désirable, la loi a voulu mettre à couvert de toute poursuite le journal qui se sera borné à présenter de ces séances un compte rendu fidèle et consciencieux» (Notice sous l'arrêt Cass., 8 février 1850, Le Siècle et Le National c. Ségur d'Aguesseau, Dalloz, 1850, I, p. 70). L'intérêt principal de cet arrêt de la Cour de cassation et son apport à l'étendue de l'irresponsabilité est d'établir jurisprudentiellement, et pour la première fois, l'intégration des comptes rendus dans le champ d'application de la protection parlementaire. La Cour décida de croiser les deux articles de loi visés : l'article 22 de la loi précitée, ainsi que l'article 11 de la loi du 25 mars 1822 , relatif au droit de réponse dans la presse. Elle rappela que le premier article «a eu pour objet de mettre les propriétaires ou gérants des journaux qui auraient rendu un compte fidèle et de bonne foi des séances publiques de la Chambre des députés à l'abri des actions pénales autorisées par la législation répressive des délits de la presse, et même des actions civiles en dommages-intérêts, à raison de ce compte rendu ». Et elle conclut grâce au second article que le droit de réponse ne se caractérise par aucune des actions précitées et donc qu'il était autorisé, même dans ce cas particulier. Enfin, le bénéficiaire reste le seul juge de la forme à donner à la réponse, étant donné qu'il s'agit d'un «droit de la défense personnelle». Ainsi, les comptes rendus publiés dans les journaux devaient bénéficier de l'irresponsabilité parlementaire. Aujourd'hui, le droit de réponse aux documents des assemblées se décline de façon différente. Ainsi, intervient la complexité du droit de réponse aux documents parlementaires parus ou reproduits sur Internet, sur le site des assemblées, par exemple. Les données sont devenues différentes, et la loi de 1881, ancienne et reprenant des dispositions de celle de 1822 , semble dépassée en ce domaine. La différence première que notent les juges, pour ce médium, est l'absence du principe de la périodicité. En effet, l'article 13 de la loi du 29 juillet 1881 envisage très clairement les «écrits périodiques ", ce qui ne peut évidemment s'appliquer à Internet. C'est ce à quoi conclut le TGI de Paris, le 5 juin 2002, par une ordonnance de référé: «attendu [...] que cet article [13] ne vise que la presse périodique; qu'en l'espèce, le requérant, qui ne prétend pas que le site litigieux soit un organe de presse, ne démontre pas le caractère périodique de ce service électronique qui par sa nature, implique, au contraire, une mise à jour continue, en tout cas, exclusive de toute périodicité» (TGI de Paris, Ordonnance de référé, 5 juin 2002 , Affaire P. H. contre S. B., req. $\mathrm{n}^{\circ} \mathrm{02} / 55659$ ). 
d'abord sur le droit de réponse qui peut être autorisé dans le cas où l'irresponsabilité ne s'étend pas aux comptes rendus publiés dans les journaux. À partir de ce droit de réponse, c'est tout le régime de la publication des comptes rendus qui en découla. Mais sous le Second Empire ${ }^{68}$, les comptes rendus devinrent « officiels» et « obligatoires». Désormais, le journaliste ne fait que reproduire intégralement un document officiel émanant de la Chambre, et logiquement, sa responsabilité ne peut être engagée s'il l'a publié fidèlement et de bonne foi. Les journaux rapportant correctement les opinions émises au sein d'une des deux Chambres ne peuvent être inquiétés. Aucune action répressive ou en dommages et intérêts n'est possible. La III ${ }^{\mathrm{e}}$ République supprime l'obligation instaurée sous le Second Empire, et fait en sorte que les Chambres communiquent rapidement leurs comptes rendus officiels ${ }^{69}$. En 1911, R. Bonnard insiste, dans la définition qu'il donne de l'irresponsabilité, sur le fait que celle-ci est une immunité « qui consiste [...] dans l'absence du droit de réponse ${ }^{70}$. Le droit actuellement en vigueur en la matière est encore celui de cette République, c'est-à-dire sous l'empire de la loi du 29 juillet 1881 . Une disposition constitutionnelle comme celle de l'article 26 de la Constitution - mais le cas se présentait déjà avec l'article 13 de la loi constitutionnelle du 16 juillet 1875 - est donc organisée par un texte à valeur législative. L'article 13 de la loi du 29 juillet 1881 reprend la rédaction exacte de l'article 11 de la loi du 25 mars 1822. Toutefois, outre cette particularité de nature juridique, le cas du droit de réponse semble aujourd'hui résolu: son impossibilité.

Cette application de l'irresponsabilité, englobant les comptes rendus des journalistes, montre bien que celle-ci ne se cantonne pas à la seule personne du parlementaire. $\mathrm{Au}$ contraire, les actes produits par un parlementaire acquièrent en eux-mêmes une protection juridictionnelle. Ils se détachent de sa personne pour être publiés par des journalistes dans des comptes rendus de séances, par exemple, et continuent à bénéficier de l'irresponsabilité parlementaire. Toutefois, cette protection générale se trouve tout de même limitée; en effet, la seule limite pourrait-on dire, outre celle de la fidélité et de la bonne foi du compte rendu, est de jouer sur la définition stricte de ce qu'est un compte rendu. Ainsi, il est légitime de penser que rapporter des débats faisant désormais partie $\mathrm{d}^{\prime}$ archives ne constitue pas un compte rendu ${ }^{71}$.

Mais le problème qui semble aujourd'hui devoir être soulevé est celui du sujet de l'irresponsabilité parlemen- taire. Faut-il toujours considérer ce dernier comme étant l'exercice des fonctions ou bien s'agit-il plutôt du lieu de cet exercice?

\section{La protection des fonctions parlementaires}

Actuellement, on assiste à une diversité des lieux de l'exercice des fonctions parlementaires (A), qui nous amène à privilégier la thèse de cet exercice comme sujet de l'irresponsabilité (B).

\section{A. La diversité des lieux de l'exercice des fonctions parlementaires}

Le système inspiré du principe séculaire rappelé par Royer-Collard: "c'est un axiome du gouvernement représentatif que la tribune n'est justiciable que de la Chambre » semble toujours être de mise. Ainsi, l'élément serait, encore aujourd'hui, éminemment spatial. C'est ce que défendent des auteurs comme O. Beaud ${ }^{72}$, reprenant la théorie pluraliste de S. Romano ${ }^{73}$. L'ordre parlementaire n'étendrait son emprise qu'au sein de l'Assemblée, sa tribune, et, on le comprend ainsi, l'enceinte de l'Assemblée nationale (ou du Sénat), afin d'inclure les commissions. L'irresponsabilité n'a cours que dans ce lieu, où s'applique l'ordre juridique parlementaire ${ }^{74}$. Ainsi, en arguant de l'article 9 de l'ordonnance $n^{\circ}$ 58-1100 du 17 novembre 1958, il serait possible, selon O. Beaud, de démontrer que l'irresponsabilité ne vaut que parce qu'elle émane de l'enceinte parlementaire. En effet, cet article dispose que "ne donneront ouverture à aucune action les discours tenus dans le sein de l'Assemblée nationale ou du Sénat ainsi que les rapports ou toute autre pièce imprimée par ordre de l'une de ces de deux assemblées». L'argument se fonde sur les termes «au sein de l'Assemblée nationale». Rappelons alors l'article 2 de cette ordonnance qui nous renseigne sur ce que comportent les locaux de l'Assemblée nationale. Celui-ci affirme que «le Palais-Bourbon est affecté à l'Assemblée nationale», sans autre mention d'aucun lieu. Ce n'est alors pas satisfaisant pour définir les différents lieux où s'exerce effectivement l'irresponsabilité de parole lorsqu'on fonde son raisonnement sur l'ordonnance de 1958. Seul l'article $1^{\mathrm{er}}$ A de l'Instruction générale du bureau

68. Résolution du 11 juillet 1848, citée par J. Poudra, Traité pratique de droit parlementaire, Paris, Quantin, 1878, p. 576.

69. Articles 4 et 5 de la résolution prise par le Bureau de l’Assemblée nationale le 26 juin 1873, et modifiée par le Bureau de la Chambre des députés le 12 août 1876 : «Un compte rendu analytique, dont l'étendue sera, au maximum, de 3 colonnes du format des grands journaux, sera mis, chaque jour et gratuitement, à la disposition des journaux de Paris et des départements », cité par J. Poudra, Traité pratique de droit parlementaire, p. 577.

70. R. Bonnard, «Immunités et discipline parlementaires... », p. 559.

71. J. Barthélemy, P. Duez, Traité de droit constitutionnel, Paris, Dalloz, 1933, p. 568: « on ne pourrait pas aller reprendre, pour les publier, dans les vieux débats parlementaires, des propos diffamatoires pour déconsidérer un adversaire. Il ne s'agit plus alors de "compte rendu".»

72. O. Beaud, «Ordre constitutionnel et ordre parlementaire...», p. 89.

73. S. Romano, «Sulla natura dei regolamenti delle camere parlamentari» [1905], in S. Romano, Scritti minori [1950], Milan, Giuffrè, 1990, p. 291, cité par O. Beaud, «Ordre constitutionnel et ordre parlementaire... », p. 88.

74. S. Romano, L'Ordre juridique, Paris, Dalloz, 1975, p. 162: «Les règlements internes des assemblées parlementaires [...] sont des éléments d'un ordre en soi, d'une institution particulière, comprise dans l'institution étatique mais distincte, et ils concernent l'institution même, en tant précisément qu'elle demeure distincte, non en tant qu'elle est absorbée dans l'institution supérieure.» 
(IGB) fait une mention différente de ce que doit comprendre l'enceinte de l'Assemblée nationale ${ }^{75}$. Néanmoins, cet article ne saurait être invoqué devant une juridiction; il faudrait retenir l'ordonnance de 1958 qui ne fait mention que du Palais-Bourbon. En effet, l'IGB n'a ni le statut, ni la valeur du règlement de l'Assemblée nationale $(\mathrm{RAN})^{76}$. L'article 61, alinéa premier, de la Constitution ne fait mention que du RAN pour le contrôle du Conseil constitutionnel, qui utilise l'ordonnance précitée comme norme de référence pour l'effectuer, et pas l'IGB. L'article 9 de cette ordonnance pourrait facilement être interprété différemment. En effet, dans sa rédaction, cet article ne fait référence «au sein de l'Assemblée nationale » qu'après le terme de " discours » et ceci, afin de bien les différencier de ceux qui seraient tenus en dehors du travail parlementaire, sur une chaîne de télévision ou lors d'un meeting politique, par exemple. Alors que les rapports, seulement évoqués plus loin dans la phrase, ne prêtent pas à confusion car il s'agit évidemment des rapports des commissions, missions d'information et délégations. Ils ne demandent donc pas cette précision car on comprend de suite qu'il s'agit d'actes de la fonction parlementaire, contrairement à des discours de députés qui pourraient être tenus ailleurs, et sans aucun rapport avec leurs fonctions.

Encore aujourd'hui, l'Assemblée nationale ne peut pas lever l'immunité de parole d'un député, mais le président de la Chambre basse, dont l'importance institutionnelle a été sauvegardée en $1958^{77}$, peut sanctionner le parlementaire auteur d'un écart de conduite. Il nous faut donc mettre en évidence la différence qui existe entre le simple député et le Président, primus inter pares. Car il est un député au caractère particulier qui ne travaille pas la plupart de son temps à la tribune - délégant souvent sa tâche d'arbitre du jeu parlementaire - ni même dans l'enceinte du Palais-Bourbon. Sous la V ${ }^{\mathrm{e}}$ République, un exemple reconnu est celui des présidents de l'Assemblée nationale, qui jouèrent un rôle très différent de celui du strict député. Ceci fut tout aussi vrai de la présidence de Jacques Chaban-Delmas à celle de Raymond Forni, dont la prétention était bien de développer, voire d'imposer une véritable «diplomatie parlementaire, en parallèle, complémentaire de celle émanant du Quai d'Orsay, et surtout plus libre ${ }^{78}$. Reste encore vrai, aujourd'hui, mais peut-être avec des variations selon les présidents, ce qu'écrivait $\mathrm{M}$. Prélot à l'aube de notre République, à savoir l'importance de «l'influence générale qui appartient au président de l'Assemblée nationale. Normalement et presque physiquement, il se trouve placé au centre de la vie politique française. Son hôtel est un lieu de visites, de rencontres, de conversations qui, incontestablement influent beaucoup sur la marche générale des affaires publiques ${ }^{79}$. La présidence de l'Assemblée nationale, l'Hôtel de Lassay, a donc bien sa spécificité spatiale - même si aujourd'hui une construction la relie au Palais-Bourbon - en plus de celles historique et politique, par rapport au lieu où travaillent les députés. Devant ce constat, il est manifeste que le véritable travail parlementaire du président de l'Assemblée nationale ne se situe pas spatialement à la tribune ${ }^{80}$, comme pour tout autre député, mais bien extérieurement à l'enceinte du Palais-Bourbon. De plus, il est évident qu'il bénéficie d'une irresponsabilité de parole, reconnue à tout député, en raison des propos qu'il pourrait y tenir, puisqu'il s'agit de l'exercice de ses fonctions. Limiter, donc, l'irresponsabilité de parole de l'ensemble des députés à la seule enceinte de l'Assemblée nationale, telle que définie par l'ordonnance de novembre 1958, semble être trop restreint. En outre, le chef de l'État peut pénétrer au sein de l'Hôtel de Lassay, comme ce fut le cas le 30 septembre 2002, alors qu'il lui est interdit de le faire au PalaisBourbon. Cela illustre que spatialement et juridiquement, les deux bâtiments sont distincts.

Désormais, les parlementaires sont de plus en plus contraints de quitter l'enceinte du Palais-Bourbon afin d'assurer leurs fonctions. Ainsi en est-il du 101, rue de l'Université qu'ils sont obligés d'occuper, vu l'exiguïté des locaux de l'Assemblée nationale. L'adresse, très proche de l'enceinte parlementaire, est tout de même différente dans sa nature intrinsèque, et la proximité n'est qu'une affaire de convenance que l'on comprend aisément. Toutefois, le fait que les députés y exercent leurs fonctions doit obligatoirement étendre l'irresponsabilité aux travaux qu'ils y accomplissent. Un exemple célèbre est la mission d'information sur le Rwanda de $1998^{81}$, au sujet du génocide perpétré dans ce pays de 1990 à 1994 . Dans cet exemple, ce sont bien les actes, lato sensu, des travaux parlementaires, allant des paroles prononcées

75. Article $1^{\text {er }}$ A de l'IGB : «L'enceinte de l'Assemblée nationale comprend : - le Palais-Bourbon ; - l'Hôtel de la Présidence ; - les immeubles du 101, rue de l'Université, du 233-235, boulevard Saint-Germain et du 32, rue Saint-Dominique. »

76. Cela est clairement illustré par le fait que l'IGB, contrairement au RAN qui «relève de ce que le droit administratif appelle les mesures d'ordre intérieur» (P. Avril, J. Gicquel, Droit parlementaire, p. 8), n'est pas contrôlée par le Conseil constitutionnel.

77. A. Martin, Le Président des assemblées parlementaires sous la $V^{e}$ République, Paris, LGDJ, 1996, p. 2: «Ce silence [de la Constitution de 1958] constitue ainsi une reconnaissance implicite de la nécessité de conserver au président des assemblées parlementaires une place non remise en cause au sein des institutions de la $\mathrm{V}^{\mathrm{e}}$ République. Alors que le constituant de 1958 procéda à une redéfinition de la notion classique du régime parlementaire, rien ne semble avoir dénaturé la substance même de la présidence des assemblées, et ceci malgré le déclassement du Parlement par rapport aux régimes antérieurs.»

78. R. Forni, France 3, le 21 novembre 2001.

79. M. Prélot, Droit parlementaire français, p. 39 .

80. On comprend ici tous les locaux pouvant servir au travail parlementaire au sein du Palais-Bourbon.

81. Mission d'information de la Commission de la Défense nationale et des Forces armées et de la Commission des Affaires étrangères, sur les opérations militaires menées par la France, d'autres pays et l'ONU au Rwanda entre 1990 et 1994. Président P. Quilès, Rapporteurs P. Brana et B. Cazeneuve, Députés. Les travaux parlementaires de cette mission, dont la réputation est très importante, est surtout «la première intrusion parlementaire», nous rappelle Albert du Roy, «dans le "domaine réservé", [qui] a permis de mettre un terme à la polémique sur la part de responsabilité française dans le génocide». Voir A. du Roy, Domaine réservé - Les coulisses de la diplomatie française, Paris, Seuil, 200o, p. 170. 
par les députés à l'imposant rapport déposé par cette Commission, et non pas des locaux supplétifs qui accordent l'irresponsabilité.

O. Beaud cite également M. Hauriou ${ }^{82}$ afin d'alléguer que l'enceinte confère l'irresponsabilité et qu'en dehors, comme dans les conseils municipaux et généraux $^{83}$, celle-ci ne pourrait trouver à s'appliquer du fait de la localisation distincte. Toutefois, ceci risque de ne plus être en phase avec la pratique; en effet, il aurait peut-être été plus opportun de citer un autre éminent professeur mais également et surtout parlementaire, comme M. Prélot ${ }^{84}$, qui écrit, une République après M. Hauriou, que ces assemblées administratives présentent une exception. Car si «le député n'est pas couvert par l'immunité au conseil général ou au conseil municipal [il n'est cependant] pas d'usage d'engager de poursuites contre lui, dans cette hypothèse ${ }^{85}$. Il s'agit donc, en l'espèce, d'une exception de fait qui infirme toute théorisation et qui ne peut donc pas lui servir de substrat. En effet, cet exemple aurait pu, juridiquement, être valable, mais la pratique l'a rendu caduc. Toutefois, s'il est possible de ne pas suivre M. Hauriou dans ce cas précis, on ne peut que lui donner raison lorsqu'il affirme que c'est l'exercice des fonctions parlementaires qui confère l'irresponsabilité.

\section{B. L'exercice des fonctions parlementaires}

En effet, lorsque M. Hauriou relève «ce lien entre l'immunité et le lieu », il précise la condition essentielle: "pour que le membre du Parlement soit couvert par l'immunité, il faut qu'il soit dans l'exercice de ses fonctions. » Et il poursuit: «en dehors de ce qui est strictement la fonction parlementaire $[\ldots]$ le député $[\ldots]$ rentre dans le droit commun ${ }^{86}$. Toutefois, il ne donne pas deux, mais trois exemples de ce que peut recouvrir la fonction parlementaire : la participation à la séance, celle aux travaux des commissions, et surtout, il conclut par la participation «aux missions parlementaires à l'extérieur ${ }^{87}$. Ainsi retranscrit, le point fondamental, en l'espèce, de la pensée de $M$. Hauriou semble être l'exer- cice des fonctions parlementaires, que celui-ci ait lieu à l'intérieur de l'enceinte parlementaire, ou bien «à l'extérieur ». Et l'exemple des missions du député à l'extérieur est très intéressant à ce niveau ${ }^{88}$. Celui-ci, totalement délaissé sous les premières décennies de la $\mathrm{V}^{\mathrm{e}}$ République, revoit le jour avec la nomination de $\mathrm{P}$. Messmer à la fonction de Premier ministre. En effet, ce dernier, lors de sa déclaration de politique générale devant l'Assemblée nationale, le 3 octobre 1972, souligne sa «volonté de mieux associer les parlementaires à l'action du gouvernement, [et décide], en accord avec le président de la République, de confier à un certain nombre de députés [...] des missions temporaires auprès des principaux ministres ${ }^{89}$. Cela signifie donc que le Gouvernement choisit de confier à des personnes, parce qu'elles sont parlementaires, des missions pour le Gouvernement. T. S. Renoux démontre très clairement que la nature parlementaire de la personne désignée par le gouvernement entre davantage en compte que ses qualités « extraparlementaires ", et qu'à ce titre, ces missions devraient être comprises dans le champ des "fonctions parlementaires ${ }^{90}$. Un exemple récent va dans le sens de cette démonstration. Ainsi, le Premier ministre, J.-P. Raffarin, a confié «une étrange $[s i c]$ mission [au député UMP D. Dord,] chargé de la valorisation des initiatives parlementaires ${ }^{91}$ afin qu'en réalité ce député modère dorénavant ses propos sur les bancs de l'Assemblée nationale. Quoi qu'il en soit, le Conseil constitutionnel considère $^{92}$ que reconnaître une irresponsabilité de parole au rapport rendu par un parlementaire en mission gouvernementale serait engendrer un régime distinct de celui prévu initialement par l'article 26 de la Constitution. Pour refuser ce nouveau régime, le Conseil fonde sa démonstration sur le terrain de l'exercice des fonctions ${ }^{93}$, et jamais ne soulève que le parlementaire travaillerait en dehors de l'enceinte parlementaire.

En définitive, on semble être arrivé à bon port avec la $\mathrm{V}^{\mathrm{e}}$ République, celle-ci reprenant une rédaction similaire à celle des deux Républiques précédentes, n’axant l'irresponsabilité ni sur la personne du député, ni sur les locaux, mais sur les travaux accomplis dans le cadre de

82. O. Beaud, «Ordre constitutionnel et ordre parlementaire... », p. 89.

83. On aurait aimé connaître le cas des conseils régionaux afin que cette démonstration soit complète, aujourd'hui. Nous ne le ferons toutefois pas ici car l'argument ne semble pas convaincant, comme nous le verrons plus loin.

84. Entre autres, professeur à la Faculté de droit de Paris, membre du Comité constitutionnel, Sénateur du Doubs, Président de la Commission du suffrage universel, des lois constitutionnelles et du règlement de l'Assemblée nationale.

85. M. Prélot, Droit parlementaire français, p. 104.

86. M. Hauriou, Précis de droit constitutionnel, Paris, Sirey, 1929, p. 533.

87. C'est nous qui soulignons.

88. Voir V. Silvera, «Les parlementaires en mission », RA, 1973, p. 409-411.

89. JOAN CR, séance du 3 octobre 1972, p. 3884.

90. T. S. Renoux, «Immunité et parlementaires... », p. 252 sq.

91. Libération, $1^{\mathrm{er}}$ octobre 2002.

92. CC Décision nº 89-262 DC du 7 novembre 1989, «Loi relative à l'immunité parlementaire», Rec., p. 90.

93. Ibid. : «Considérant que les députés auteurs de la saisine font valoir que la loi relative à l'immunité parlementaire étend l’irresponsabilité instituée par le premier alinéa de l'article 26 de la Constitution à des actes autres que les opinions ou votes émis par un parlementaire dans l'exercice de ses fonctions; qu'en effet, la rédaction d'un rapport, à la demande ou pour le compte du Gouvernement, diffusé sur son ordre, ne semble pas pouvoir être rattachée à l'exercice du mandat parlementaire; que l'article LO 144 du code électoral qui prévoit l'éventualité pour un député d'accomplir une mission temporaire à la demande du Gouvernement a pour seul objet de déroger pour une période de six mois à une incompatibilité » et qu'ainsi, «la mission qu'exerce un député ou un sénateur à la demande du Gouvernement ne s'inscrit pas dans l'exercice de sa fonction parlementaire». 
l'exercice des fonctions parlementaires. H. Bonneau est déjà très clair en 1948 lorsqu'il écrit que «l'irresponsabilité joue pour tous les actes de la fonction et il n'y a pas lieu de distinguer à ce sujet les opinions et votes émis dans les commissions de ceux qui se sont manifestés au sein des assemblées elles-mêmes ${ }^{94}$. Il marque bien une différence entre le «sein des assemblées » et le lieu où se réunissent les commissions. Défendre l'idée selon laquelle ce sont les locaux qui octroient une immunité aux parlementaires serait dangereux et contreviendrait au principe perpétué lors des Républiques précédentes, en étendant les exceptions au principe d'égalité des citoyens devant la loi ${ }^{95}$.

Récemment, la Cour européenne des droits de l'homme (CEDH) a semblé clore la discussion, en allant jusqu'à consacrer juridiquement la pratique que relevait M. Prélot sous la IV ${ }^{\mathrm{e}}$ République. Par sa jurisprudence Jerusalem c. Autriche $e^{96}$, la CEDH avait à connaître d'une espèce dans laquelle $\mathrm{M}^{\mathrm{me}}$ Jerusalem, membre d'un conseil municipal, et ne bénéficiant à ce titre d'aucune irresponsabilité, était attaquée pour avoir tenu des propos que d'aucuns pouvaient qualifier d'injurieux et dénués de fondement. $\mathrm{M}^{\mathrm{me}}$ Jerusalem est également membre du parlement du Land et bénéficie à cette occasion d'une irresponsabilité. Les propos attaqués ont été prononcés lors d'une séance du conseil municipal au sujet d'une «question politique». La CEDH «rappelle que, précieuse pour chacun, la liberté d'expression l'est tout particulièrement pour un élu du peuple: il représente ses électeurs, signale leurs préoccupations et défend leurs intérêts ", telle est la mission du mandat parlementaire, qui se manifeste par les actes de la fonction parlementaire. À ce propos, la solution de la CEDH est univoque : «La Cour relève [que les propos en cause] ont été tenus au cours d'un débat politique du conseil municipal [...]. Le fait que ce débat ait eu lieu devant le conseil municipal [et non le parlement du Land] n'est pas décisif. Que les propos de la requérante aient été ou non couverts par l'immunité parlementaire, la Cour estime qu'ils ont été prononcés dans une instance pour le moins comparable au Parlement pour ce qui est de l'intérêt que présente, pour la société, la protection de la liberté d'expression des participants»; et de conclure: «dans une démocratie, le Parlement ou des organes comparables sont des tribunes indispensables au débat politique.» Ainsi, la $\mathrm{CEDH}$ semble avancer un début de réponse à la récente interrogation de G. Bergougnous: "amputée dans sa portée, souvent contestée, comme l'attestent des contentieux récents ${ }^{97}$ mettant en cause les propos ou les écrits de parlementaires, l'irresponsabilité résistera-t-elle à un mouvement qui a déjà conduit à réformer l'inviolabilité $^{98}$ afin de l'adapter aux exigences actuelles? " ${ }^{99}$. Selon la Cour européenne, la réponse existe et elle est négative si on doit entendre par-là une limitation de l'étendue de l'irresponsabilité. Car la $\mathrm{CEDH}$ montre une volonté d'intégrer sous un régime d'irresponsabilité tout acte, relevant de la liberté d'expression de l'élu, dans l'exercice de ses fonctions politiques. Et plutôt qu'une remise en cause de l'objet de l'irresponsabilité, il semble que ce soient des aménagements qui deviennent nécessaires.

\section{Les aménagements nécessaires}

Le règlement de l'Assemblée nationale apporte des limites à l'irresponsabilité parlementaire prévue par la Constitution (A), sans toutefois présenter une solution satisfaisante à certaines circonstances très problématiques (B).

\section{A. Les limites disciplinaires à l'irresponsabilité constitutionnelle}

«Les Assemblées ne pourraient subsister si elles n'avaient un droit de discipline sur leurs membres ${ }^{100}$. Cette affirmation solennelle formulée par E. Pierre montre bien l'importance pour les Chambres de disposer d'un droit de discipline, interne et autonome. «Ce privilège qui est d'ordre supérieur, n'a pas moins d'importance que celui qui soustrait à tout jugement extérieur les paroles et les votes $"{ }^{101}$; et celui-ci est bien évidemment organisé par le règlement de l'assemblée parlementaire. C'est son droit de police interne. Du fait de sa nature, le régime disciplinaire de l'Assemblée ne vient pas, contrairement à la plupart des articles du RAN, compléter dans les détails des dispositions constitutionnelles, mais s'inscrit tout de même dans la logique de la séparation des pouvoirs.

L'irresponsabilité doit couvrir toute opinion émise par le député dans l'exercice de ses fonctions. Néanmoins, la Chambre basse sait parfois limiter cette immunité absolue en imposant une certaine morale. Ainsi, en 1986, J.-C. Martinez, alors député, s'est illustré par un discours, en tant que rapporteur spécial pour la commission des finances, dont «le ton $[. .$.$] employé était déjà très polé-$ mique [...] et a suscité des réserves [...] de membres tant de l'opposition que de la majorité ${ }^{102}$. Dans ce cas précis, il n'était pas encore question d'une quelconque censure à

94. H. Bonneau, «Immunités parlementaires dans la Constitution française du 27 octobre 1946 », RDP, 1948, p. 62.

95. À ce sujet, la Cour de cassation affirme que cette irresponsabilité doit être entendue dans le sens le plus strict en raison de son caractère exceptionnel. Voir par exemple : Cass. crim., 22 février 1956, Général Revers c. Varin, Gazette du Palais, 1956, Jurisprudence, p. 375.

96. CEDH, 27 février 2001, Jerusalem c. Autriche, req. $n^{\circ} 26958 / 95$.

97. Ceux cités par G. Bergougnous sont antérieurs à l'affaire Jerusalem c. Autriche.

98. C'est-à-dire à sa limitation.

99. G. Bergougnous, «Le statut de parlementaire... », p. 344

100. E. Pierre, Traité de droit politique..., p. 505.

101. Ibid., p. 1273.

102. JOAN CR, deuxième séance du 3 novembre 1986, p. 5696. 
son égard, mais le président de la Commission décida tout de même de lui rappeler que les députés «jouiss[aient] de l'immunité parlementaire pour [leurs] écrits ou [leurs] propos dans le cadre de l'Assemblée nationale, mais que cela [leur] imposait d'autant plus un devoir de réserve ${ }^{103}$. Le président de la Commission des finances ajouta qu'il jugeait certains propos «inacceptables », et laissa le député devant sa conscience. Celui-ci décida alors, presque de son propre chef, de modifier certains des termes de son discours à joindre au rapport de la Commission. Mais il ne s'agit ici que d'une conséquence de l'autorité morale de la Commission parlementaire, alors que celle-ci, dans sa majorité, semblait vouloir interdire certains propos, propres à mettre en cause l'autorité, la réputation de la Commission, voire, par prolongement, de l'Assemblée elle-même. Dans ces conditions, il apparaît problématique que les paroles prononcées soient obligatoirement couvertes par l'irresponsabilité alors qu'elles sont désavouées par une large majorité des parlementaires. Sous la III ${ }^{\mathrm{e}}$ République déjà, J. Barthélemy et P. Duez évoquaient l'idée d'une limite à cette immunité absolue: le cas de faute lourde. Ainsi, écrivent-ils, «en protégeant les parlementaires contre les actions intentées avec intention malicieuse, on pourrait laisser les parlementaires responsables de leurs fautes lourdes et de leurs imprudences inexcusables ${ }^{104}$.

Aujourd'hui, du fait de son droit de discipline autonome, l'Assemblée nationale peut limiter l'irresponsabilité prévue par la Constitution pour «des actes parlementaires excessifs ${ }^{105}$. Le paragraphe 4 de l'article $73 \mathrm{du}$ RAN dispose en effet que "la censure avec expulsion temporaire du Palais de l'Assemblée nationale est prononcée contre tout député: qui s'est rendu coupable d'injures, provocations ou menaces envers le président de la République, le Premier ministre, les membres du Gouvernement et les assemblées prévues par la Constitution». La mesure est très grave car elle interdit au député sanctionné de réapparaître dans les locaux parlementaires avant quinze jours. Il semble donc qu'il faille atténuer l'affirmation de M. Hauriou selon laquelle «il faut que toute vérité, même diffamatoire ou injurieuse, pour des personnalités déterminées, puisse être dite publiquement ${ }^{106}$. Les assemblées parlementaires ne sont pas des lieux où il serait autorisé de traîner quelqu'un sur la claie; en effet, que ce soit historiquement en Angleterre ou bien en France, les "personnalités » - c'est-àdire les personnes visées expressément, nommées - ont l'interdiction d'être mentionnées. E. Pierre, dans son Traité de droit politique, électoral et parlementaire, le rappelle très clairement par un précédent univoque dans lequel le Président Grévy explique à un député que «les personnalités ne doivent pas être portées à la tribune ${ }^{107}$. De plus, le RAN impose cette limite au sujet des questions écrites ${ }^{108}$. Certes, l'immunité contre les poursuites pénales et civiles court toujours, mais il faut se rendre compte que le député ne pourra pas très longtemps être repris constamment à l'ordre par le président de l'Assemblée nationale et sanctionné par ses collègues. Cette situation ne peut raisonnablement se prolonger dans le temps. Car les peines se cumulent ${ }^{109}$ : exclusion temporaire ${ }^{110}$, privation de la moitié de l'indemnité pendant six mois et jusqu'à la saisine du procureur général par le président de l'Assemblée nationale.

Le passage de l'article 73 relatif au président de la République illustre le fait que l'on ne puisse citer de " personnalités » ${ }^{111}$ à la tribune, et permet également de s'apercevoir que dans ce cas, une réflexion doit être menée par les parlementaires, et sans doute par le constituant. En effet, selon l'article 73 du RAN, un député a l'interdiction d' «injurier» ou de "provoquer» le président de la République à la tribune. Cette limitation peut se révéler importante puisque les députés "fautifs" ne sont pas maîtres de l'interprétation de leurs paroles, cela échéant au président de l'Assemblée nationale, dans un premier temps, puis à la Chambre dans son ensemble. Le rappel à l'ordre n'est pas systématique de la part du président de l'Assemblée nationale, comme l'illustre l'événement survenu le 12 novembre 1980 lorsque G. Fillioud eut proféré des propos que l'on pouvait estimer injurieux à l'égard du président de la République, V. Giscard d'Estaing ${ }^{112}$. La seule «sanction» fut que le Premier ministre de l'époque, R. Barre, demanda au président de

103. JOAN CR, deuxième séance du 3 novembre 1986, p. 5696.

104. J. Barthélemy, P. Duez, Traité de droit constitutionnel, p. 569.

105. J. Gicquel, Droit constitutionnel et Institutions politiques, p. 642.

106. M. Hauriou, Précis de droit constitutionnel, p. 533.

107. Épisode rapporté, lors de la séance du 5 novembre 1878 à la Chambre des députés, par E. Pierre, Traité de droit politique..., note 3, p. 517 : « M. le Président Grévy - Monsieur de Cassagnac, vous ne devez pas porter à la tribune, contre une personne qui n'appartient pas à la Chambre, qui n'est pas ici pour se défendre, des suppositions de cette nature, dont vous comprenez toute la vérité. M. Paul de Cassagnac-Quand je parle d'un député, M. le Président me dit : il ne faut pas parler d'un collègue! Quand je parle de toute autre personne, il ne faut pas parler d'une personne étrangère. Je voudrais bien savoir de qui je puis parler. M. le Président-Ce qui revient à dire que les personnalités ne doivent pas être portées à la tribune, et que, ces personnalités, on doit surtout se les interdire lorsqu'elles revêtent la forme d'imputations de la nature de celles que vous dirigez contre le sous-préfet dont vous parliez.»

108. Article $139,2^{\circ}$ du RAN.

109. Article 77 du RAN.

110. Article 74 du RAN : «... le député est reconduit jusqu’à la porte du Palais par le chef des huissiers.»

111. Au sens défini précédemment.

112. JOAN CR, première séance du 12 novembre 1980, p. 3762 : «M. Georges Fillioud - Vous ne vouliez pas, monsieur le garde des Sceaux, qu'on parle des diamants offerts par Bokassa à Giscard. [...] Vous ne vouliez pas qu'on établisse le lien entre ces donations et l'emprisonnement par lettre de cachet de Delpey coupable d'en détenir la liste. Ce lien est maintenant évident! Après ces erreurs, vous avez commis un crime: celui de forfaiture en soustrayant des pièces au dossier confié à la cour de sûreté de l'État parce que ces pièces étaient compromettantes pour le président de la République et sa famille. » 
l'Assemblée de bien vouloir accepter que le garde des Sceaux ne répondît pas, "étant donné les termes utilisés ${ }^{113}$.

Toutefois, l'article 73 peut tout de même être mis en œuvre; pour la première fois sous la $\mathrm{V}^{\mathrm{e}}$ République, trois députés, de l'opposition, ont été sanctionnés par une censure simple en 1984. Les députés, MM. Toubon, d'Aubert et Madelin, étaient accusés d'avoir tenu, d'après le président de l'Assemblée nationale, Louis Mermaz, des propos injurieux à l'encontre du président de la République, F. Mitterrand ${ }^{114}$, relatifs au passé de celui-ci dans la Résistance. Ces députés refusant de retirer leurs propos, après avoir eu l'occasion de les expliquer publiquement, ont été sanctionnés par un vote « assis debout », en violation avec les dispositions du RAN ${ }^{115}$ (qui impose une censure avec exclusion) alors que le président de l'Assemblée a pour fonction de le faire respecter ${ }^{116}$. Sans doute celui-ci ne voulait-il alors pas être taxé de procureur politique par une partie significative de l'Hémicycle. En tout cas, il s'agit bien en l'espèce d'une sanction morale - terme présent pendant tout ledit débat - comme le reconnaît le Président Mermaz ${ }^{117}$. À ce propos, il ne s'agit pas d'une nouvelle portée donnée à la sanction : un précédent à la Chambre basse était déjà intervenu dans ce sens. Ainsi, lors de la séance du 9 février 1831, le Président Casimir-Perier déclare « que le Président n’a qu'une force morale pour maintenir l'ordre et faire respecter le règlement. Les députés qui violent le règlement et ne font aucun cas des observations du Président manquent à la dignité de la Chambre. Ils se manquent à eux-mêmes ${ }^{118}$.

Le précédent de 1984 illustre bien que les députés ne peuvent mettre en cause le président de la République dans l'exercice de leurs fonctions sans encourir de sanction disciplinaire. Toutefois, situation frappante, le Premier ministre, quant à lui, peut mettre en cause le chef de l'État au sein de l'Hémicycle, devant la représentation nationale, sans être inquiété par une quelconque procédure réglementaire. C'est ce qui se déroula lors de la séance des questions d'actualité du 13 juin 2001 pendant laquelle Lionel Jospin a déclaré avoir «tardé à [s]'expliquer devant les journalistes. C'est tout de même moins grave que de tarder à s'expliquer devant les juges » ${ }^{119}$. Par ces paroles, le Premier ministre, nommé par le président de la République, attaque implicitement celui-ci. Entre les députés et les ministres, se présente un décalage de situation, en défaveur des parlementaires, alors même qu'ils siègent dans leur assemblée. Le premier remède qui vient à l'esprit est radical: la suppression de la limite posée par l'article 23 de la Constitution. Ceci aurait en outre la vertu de renforcer les assemblées. P. Avril l'évoque $^{120}$, A. Le Divellec l'affirme: «La "revalorisation” du Parlement français passera donc moins par l'accroissement de ses compétences formelles [...] que par la suppression de l'incompatibilité, qui ne présente aucun avantage sérieux, mais contribuerait certainement à favoriser l'influence réelle de la majorité sur le Gouvernement ${ }^{121}$. Et quand bien même; une seconde « révolution» s'imposerait alors: lever l'impossibilité, déjà présente sous la III ${ }^{e}$ République, d'infliger une sanction disciplinaire à un ministre. On comprend aisément que la situation actuelle semble lointaine. Et la seule solution possible, partiellement dans les mains des députés, consiste finalement à revenir à la cause de l'absence, pendant longtemps, de peines disciplinaires dans le RAN : «la bonne éducation " ${ }^{122}$ en politique. Sans cet effort, il s'avère très probable que les députés restent dans cet état de différence de traitement.

\section{B. L'écart de situations entre l'Hémicycle et son perron}

Il est des cas où la situation devient des plus confuses. De nombreux députés ont été indignés par des événements qui se sont déroulés à l'Assemblée nationale lors de la session 1999-2000. Ainsi, R. Forni, qu'on a pu qualifier de «président-partisan » ${ }^{123}$ de l'Assemblée nationale et non pas arbitre, sanctionne N. Mamère, membre de la majorité parlementaire, par application de l'article $73 \mathrm{du}$ RAN, pour les propos « sévères » qu'il tint à l'encontre du chef de l'État dans l'Hémicycle. En l'espèce, R. Forni juge que le RAN est violé et affirme « de la façon la plus ferme, qu['il] considère [les propos de $\mathrm{M}$. Mamère] comme inacceptables. Ils sont contraires à toute la tradition républicaine régissant le fonctionnement de notre assemblée,

113. JOAN CR, première séance du 12 novembre $1980, \mathrm{p} .3763$.

114. JOAN CR, troisième séance du 2 février 1984, p. 475 sq.

115. Article 73 du RAN.

116. Et L. Mermaz le disait pourtant lui-même: «... je serai obligé [...] de consulter l'Assemblée sur une sanction éventuelle, en application du règlement ", JOAN CR, troisième séance du 2 février 1984, p. 478.

117. Ibid., p. 481 : «En votant la censure, nous avons porté une condamnation morale.»

118. Précédent rapporté par E. Pierre, Traité de droit politique..., p. 515.

119. JOAN CR, première séance du 13 juin 2001, p. 4163. On peut toutefois noter que L. Jospin, une fois devenu Premier ministre, revient sur l'argumentation qu'il développait lors de la séance du 2 février 1984, à savoir que l'on ne peut mettre en cause le président de la République au sein de l’Hémicycle parce que celui-ci ne "peut répondre à aucun de ceux qui, dans cette assemblée, l’ont mis en cause! », JOAN CR, troisième séance du 2 février 1984, p. 479.

120. P. Avril, «Réflexions sur l’incompatibilité édictée par l’article 23 de la Constitution », in Mélanges Gélard, Paris, Montchrestien, 2000, p. $145-149$.

121. A. Le Divellec, «La chauve-souris », in Mélanges Avril, Paris, Montchrestien, 2001, p. 359-360.

122. J. Barthélemy, P. Duez, Traité de droit constitutionnel, p. 558.

123. "Devant ce bilan, devant ce retour de l'ambition politique, les attaques de l'opposition sont affligeantes et parfois même pathétiques", écrivait-il dans Le Monde, 16 juin 2001, p. 1. De plus, A. Montebourg, député PS, attaquait la position «inacceptable » et «hautement contestable» de R. Forni qui faisait «pression sur les députés pour qu'ils ne signent pas [sa] proposition de résolution de renvoi de Jacques Chirac devant la Haute Cour de Justice", et qui expliquait "au mépris du règlement, qu'il refuser[ait] d'inscrire la proposition à l'ordre du jour même si les 58 signatures sont réunies ", Le Monde, 28 juin 2001, p. 7. 
selon laquelle le chef de l'État ne saurait, dans cette enceinte, de quelque manière que ce soit, faire l'objet d'imputation à caractère personnel » ${ }^{124}$. Le RAN et la tradition de l'Assemblée nationale interdisent de mettre en cause une "personnalité». Cela est vrai à l'égard du président de la République tout comme d'un autre député, comme le rappelle R. Forni à N. Mamère: "Quant aux propos tenus à l'encontre de celui de nos collègues qui a exercé la fonction qui est aujourd'hui la mienne, ils constituent une attaque personnelle inadmissible au regard de notre règlement. » Cette remarque fait référence aux attaques portées à l'encontre de $\mathrm{P}$. Séguin ${ }^{125}$, visé tout comme le chef de l'État par les insinuations de N. Mamère pendant la séance de questions d'actualité ${ }^{126}$. La motivation du président de l'Assemblée, en sanctionnant le député Vert, fut de préserver « la limite qui touche au respect des personnes et de la fonction [parlementaire] $\gg{ }^{127}$. L'irresponsabilité, justifiée par l'exercice de la fonction parlementaire, trouve donc une limite réglementaire dans le respect de cette même fonction.

Quoiqu'il en soit, N. Mamère fut sanctionné pour ses propos tenus dans l'enceinte parlementaire. Mais lors de sa sortie de l'Hémicycle, le député ne se priva pas de poursuivre son discours « offensif» devant les journalistes ${ }^{128}$, sachant pertinemment que la règle traditionnelle de l'Élysée est de ne jamais porter plainte pour diffamation ${ }^{129}$. Dans la même situation, on peut citer A. Montebourg qui écrivait que «se taire sur de telles pratiques est impossible, puisqu'elles sont un fait et une vérité cruelle que seuls les partisans embarrassés du président de la République ne peuvent pas et ne veulent pas entendre ${ }^{130}$. La situation est singulière: pendant que ces députés sont dans l'exercice de leurs fonctions, ils sont sous le couvert de l'irresponsabilité, mais également sous l'empire des limites réglementaires. Alors qu'une fois hors de leurs fonctions, de fait, ils peuvent librement s'exprimer sur le perron du Palais-Bourbon.

La deuxième singularité procède directement des propos tenus par R. Forni, alors président de l'Assemblée nationale. Ainsi, sur le même sujet, les fraudes électorales et la Mairie de Paris, celui-ci déclare lors d'émissions radiodiffusées que la protection pénale du chef de l'État provient d'une «décision assez curieuse du Conseil constitutionnel» et qui "était contestée par tous, sauf par ceux qui $[y]$ ont intérêt» ${ }^{131}$. R. Forni déclarait également: «que ce système [RPR] ait été organisé de manière scientifique dans une collectivité qui s'appelle la mairie de Paris, ça me paraît évident ${ }^{132}$ [et] le président rendrait un grand service en allant s'expliquer [... devant la justice » ${ }^{133}$. Ou bien, lors d'une autre émission radiodiffusée, il ajoute, au sujet du «système Chirac», qu' " on est toujours l'héritier de quelqu'un. [...] Tiberi est devenu maire de Paris après Jacques Chirac et, si j'entends bien ce qui se dit, les affaires remontent à il y a longtemps; sans doute y avait-il une espèce d'institutionnalisation, un système ${ }^{134}$. Ainsi, le président de l'Assemblée nationale tient les mêmes propos / insinuations / mise en cause du chef de l'État que le député qu'il avait sanctionné peu de temps auparavant. Autre signe du décalage de la situation, une fois hors de l'enceinte parlementaire, ce sont les trois présidents de groupes de l'opposition qui rappellent à l'ordre le président de l'Assemblée nationale, "lui adressant une lettre commune pour l'appeler au "respect" du chef de l'État» ${ }^{135}$. Toujours abrité par la même tradition selon laquelle le président de la République n'intentera aucune action en diffamation, R. Forni n'hésite pas à multiplier ses accusations publiques à l'encontre du chef de l'État, sans être inquiété. Situation d'autant plus cocasse que la Cour de cassation, en 1988, a rendu un arrêt lui expliquant les limites de sa protection ${ }^{136}$ : étant entendu qu'elle n'a pas cours dans ces circonstances. La Cour argumente de la façon suivante: «Les propos imputés à Raymond Forni n'ont pas été tenus au cours de l'une des activités prévues aux titres IV et V de la Constitution, pouvant seuls caractériser l'exercice des fonctions parlementaires, non plus qu'au sein de l'Assemblée nationale ${ }^{137}$. Si la solution de cet arrêt est légitime, ses motifs n'en restent pas moins critiquables à plusieurs égards. Tout d'abord, ce n'est pas le sein de l'Assemblée nationale qui octroie l'irresponsabilité mais les fonctions parlementaires ; ensuite, celles-ci ne sont pas celles du Parlement (Titres IV et V), comme

124. JOAN CR, séance du 31 mai 2000, p. 4794.

125. Règle toujours aussi vivace, selon laquelle on ne met pas en cause un autre député, puisque J.-L. Debré a repris - sûrement sur la base de l'article 71 , $5^{\circ}$ du RAN - Y. Lachaud, membre de la majorité parlementaire, pour avoir déclaré: «... M. Liberti, qui semble mal connaître le Gard... », lors de la séance du 9 octobre 2002. http://www.assemblee-nat.fr/12/cra/2002-2003/011.asp\#P52_1094.

126. «Les quinze personnes qui ont été récemment mises en examen par la justice [...] sont la partie immergée d’un iceberg beaucoup plus important, ce qui montre que cette fraude électorale a été organisée au plus haut niveau. [...] Derrière tout cela, sont concernées des personnalités au sommet de l'État. Je rappelle que l'actuel président de la République était maire de Paris », JOAN CR, deuxième séance du 30 mai 2000 , p. 4736.

127. JOAN CR, séance du 31 mai 2000, p. 4794

128. Le Monde, 2 juin 200o, p. 6.

129. L'article 26 de la loi du 29 juillet 1881, le délit d'offense, n'est jamais invoqué par l'Élysée.

130. Le Monde, 7 juin 2000, p. 17.

131. Le Monde, 10 octobre 2000, p. 8. Le Conseil constitutionnel a alors estimé qu'il lui fallait, fait unique, publier un communiqué officiel, dès le 10 octobre, afin d'expliciter la décision critiquée.

132. Le Monde, 10 octobre 2000, p. 8.

133. Le Monde, 11 octobre 2000, p. 8.

134. Le Monde, 27 juin 2000, p. 18.

135. M.-B. Allaire, P. Goulliaud, L'Incroyable septennat, Paris, Fayard, 2002, p. 524.

136. Cass. crim., 7 mars 1988, Forni, La Semaine juridique, 1988, II, J, 21132-21133.

137. Ibid. 
le démontre T. S. Renoux. Enfin, cet arrêt illustre la rigidité du système français. Par exemple, celui retenu par la Suisse est plus souple et s'exonère du caractère absolu de l'irresponsabilité, véritable "carcan parlementaire» devenu obsolète plus de deux cents ans après sa mise en place en France. Il est à espérer que les députés soient depuis devenus des majeurs politiques, capables d'utiliser les techniques parlementaires britanniques sans contraintes juridiques. Le système suisse pourrait être une inspiration car il dispose que les juges demandent l'autorisation des assemblées afin de pouvoir entamer des poursuites, l'irresponsabilité y ayant un champ d'application très large. De la sorte, en 1970, un député helvète répéta des renseignements militaires secrets, révélés devant le Conseil national, dans un journal ainsi qu'à un expert. Le code pénal suisse protège la publication faite dans le journal! Quant à la révélation faite à l'expert, «l'autorisation de poursuivre fut demandée aux Chambres, qui la refusèrent [, estimant] que le comportement [du député], assurément peu discret, avait jeté une lumière utile sur un secteur de la défense nationale dont l'Administration fédérale n'avait pas suffisamment montré les faiblesses ${ }^{138}$.

Actuellement, en France, les députés peuvent librement faire des révélations dans un rapport parlementaire - dont la diffusion et la lecture sont encore souvent confidentielles - sans être inquiétés ; mais cela est impossible par la voie des médias, audiovisuels par exemple. Les députés qui interviennent publiquement sont souvent des présidents de commissions parlementaires ${ }^{139}$, et à ce titre, ils devraient pouvoir rendre compte de leurs informations devant le nombre le plus large de citoyens, et non plus seulement pour les cénacles politiques et universitaires. La Cour d'appel de Paris a récemment rendu une décision dans ce sens. En l'espèce, le député J. Guyard avait été condamné le 21 mars 2000 par le TGI de Paris pour diffamation publique: il avait qualifié le mouvement anthroposophe de «secte» lors d'un journal télévisé de France 2, le 17 juin 1999, sans pouvoir argumenter, car des renseignements relevaient du secret du travail de la commission. Dans son arrêt du 6 septembre $2001^{140}$, la Cour relève que des développements critiques et justifiés, relatifs à ce mouvement, sont bien présents dans le rapport parlementaire. Certes, le juge applique le droit en vigueur, relève que l'irresponsabilité ne s'étend pas aux paroles de J. Guyard et affirme que «ces commentaires présentent bien un caractère diffamatoire». Néanmoins, la Cour d'appel conclut que « Jacques Guyard a été convié à l'émission en sa qualité de président de la Commission sur les sectes, qu'il s'est exprimé en tant que porte-parole d'un travail collectif; qu'il ne pouvait y avoir sur ce point aucune ambiguïté dans l'esprit des téléspectateurs; que Jacques Guyard s'est borné à reprendre des accusations figurant dans un rapport officiel; que ses propos ne dénaturent pas le contenu du rapport; que ni les termes utilisés, ni le ton employé ne manifestent une quelconque animosité personnelle». Par conséquent, les juges relaxent le député sur l'excuse de la bonne foi, à la manière de l'auteur d'un compte rendu publié dans un journal. Mais il faut surtout y voir une solution jurisprudentielle, en phase avec l'évolution des fonctions des députés, prenant note que «le cour de la vie politique nationale» n'est peut-être plus seulement le Parlement, mais en partie les médias. Finalement, on ne peut que se rendre compte que le statut de parlementaire «n'a jamais véritablement fait l'objet d'une réflexion d'ensemble visant à redéfinir les droits et obligations des représentants de la Nation » ${ }^{141}$, que celle-ci devient nécessaire, et surtout que ce n'est pas aux juges de pallier son absence.

D’une manière générale, le pouvoir devrait être reconnu à une Assemblée nationale émancipée de décider si un député se trouve dans la situation de voir sa liberté d'expression protégée ou non. La condition devrait être la même que celle retenue clairement par la $\mathrm{CEDH}$, à savoir : un lien étroit entre les propos du parlementaire et l'exercice de ses fonctions stricto sensu ${ }^{142}$. Ainsi, la Cour estime, lors d'une affaire relative à « un droit à la protection de sa réputation», que le droit d'accès à un tribunal (article $6 \$ 1 \mathrm{CEDH})$ «n'est pas absolu, mais peut donner lieu à des limitations implicitement admises » ${ }^{143}$. De plus, « la Cour relève que le fait pour les États d'accorder généralement une immunité plus ou moins étendue aux parlementaires constitue une pratique de longue date, qui vise à permettre la libre expression ${ }^{144}$ des représentants du peuple et à empêcher que des poursuites partisanes puissent porter atteinte à la fonction parlementaire». De même, la Cour avait estimé quelques mois auparavant que: "the parliamentary immunity enjoyed by the MP in the present case pursued the legitimate aims of protecting free speech in Parliament and maintaining the separation of powers between the legislature and the judiciary ${ }^{145}$. La CEDH effectue, en la matière, un contrôle approfondi, non pas seulement en examinant « in abstracto la législation et la pratique pertinentes, mais [en recherchant également] si la manière dont elles ont touché le requérant a enfreint la Convention ${ }^{146}$. Comme le note J.-F. Flauss, ces espèces «sont illustratives d'un renforcement du contrôle européen sur la mise en œuvre de l'immunité parlementaire et plus généralement de toute immunité comparable, et partant

138. J.-F. Aubert, Supplément 1967-1982, Neuchâtel, Ides et Calendes, 1982, p. 141.

139. Ce fut le cas de R. Forni en 1988 et de J. Guyard en 2001.

140. CA Paris, 6 septembre 2001, Jacques Guyard, req. $\mathrm{n}^{\circ}$ oo / 02776.

141. G. Bergougnous, «Le statut de parlementaire... », p. 361.

142. CEDH, 30 janvier 2003, Cordova c. Italie $\left(n^{\circ} 1\right)$, req. $40877 / 98$.

143. Ibid.

144. C'est nous qui soulignons.

145. CEDH, 17 décembre 2002, A. v. The United Kingdom, req. 35373/ 97.

146. $\mathrm{CEDH}, 3$ janvier 2003, Cordova c. Italie ( $\left.\mathrm{n}^{\circ} 1\right)$, req. $40877 / 98$. 
d'une nouvelle érosion de l'autonomie constitutionnelle des États ». Mais l'auteur ajoute immédiatement qu'elles sont « loin d'être révolutionnaires » car « elles préservent [...] le droit des États d'établir, au profit des autorités constitutionnelles, une immunité juridictionnelles civile revêtant un caractère absolu » ${ }^{147}$.

La CEDH laisse donc une marge importante aux États contractants pour organiser l'irresponsabilité de leurs parlementaires nationaux ${ }^{148}$. La France n'étant pas la plus protectrice et la Cour se référant aux canons européens afin d'apprécier les limites de l'irresponsabilité, une réforme est donc envisageable afin de renforcer la protection dont jouissent actuellement les députés français. On pourrait ainsi profiter de l'occasion prochaine de la révision constitutionnelle relative au statut pénal du chef de l'État pour y joindre une réforme de l'irresponsabilité parlementaire. Et s'il fallait proposer une piste de réflexion, on pourrait dire de l'Italie qu'elle pré- sente aujourd'hui un système très intéressant. Ce pays autorise qu'un juge judiciaire, estimant que les propos tenus par un parlementaire ne peuvent manifestement pas se rattacher à l'exercice de ses fonctions, peut condamner ce parlementaire, ou surseoir à statuer, le temps de demander l'avis de l'assemblée dont il relève. La Cour constitutionnelle italienne interdit effectivement aux juges d'entamer ou de continuer des poursuites contre un parlementaire alors que son assemblée aurait voté le maintien de son irresponsabilité, estimant que les propos en cause ont un lien avec l'exercice des fonctions parlementaires. La Chambre des députés a élargi le champ d'application de l'irresponsabilité aux propos tenus en dehors de l'enceinte parlementaire; s'ils ont un lien étroit avec l'exercice de la fonction parlementaire, a récemment précisé la Cour constitutionnelle italienne. Ce système aurait permis à J. Guyard de ne pas être inquiété par la justice française. 